\title{
Evaluating blood-brain barrier permeability in a rat model of type 2 diabetes
}

\author{
Ju Qiao' ${ }^{1}$, Christopher M. Lawson ${ }^{1}$, Kilian F. G. Rentrup ${ }^{1}$, Praveen Kulkarni ${ }^{1}$ and Craig F. Ferris ${ }^{1,2^{*}}$ (D)
}

\begin{abstract}
Background: This is an exploratory study using a novel imaging modality, quantitative ultrashort time-to-echo, contrast enhanced (QUTE-CE) magnetic resonance imaging to evaluate the permeability of the blood-brain barrier in a rat model of type 2 diabetes with the presumption that small vessel disease is a contributing factor to neuropathology in diabetes.

Methods: The BBZDR/Wor rat, a model of type 2 diabetes, and age-matched controls were studied for changes in blood-brain barrier permeability. QUTE-CE, a quantitative vascular biomarker, generated angiographic images with over 500,000 voxels that were registered to a 3D MRI rat brain atlas providing site-specific information on blood-brain barrier permeability in 173 different brain areas.

Results: In this model of diabetes, without the support of insulin treatment, there was global capillary pathology with over $84 \%$ of the brain showing a significant increase in blood-brain barrier permeability over wild-type controls. Areas of the cerebellum and midbrain dopaminergic system were not significantly affected.

Conclusion: Small vessel disease as assessed by permeability in the blood-brain barrier in type 2 diabetes is pervasive and includes much of the brain. The increase in blood-brain barrier permeability is a likely contributing factor to diabetic encephalopathy and dementia.

Keywords: Quantitative ultrashort time-to-echo, Contrast enhanced (QUTE-CE), Magnetic resonance imaging, Small vessel disease, BBZDR/Wor rat, Diabetic encephalopathy, Vascular biomarker, Ferumoxytol
\end{abstract}

\section{Background}

Vascular dementia is a serious consequence of diabetes [1]. Prolonged exposure to high blood levels of glucose, typical of type 2 diabetes, affects capillary endothelial structure, function and permeability [2]. Failure in the blood brain barrier lies at the foundation of cerebral small vessel disease and contributes to the pathogenesis of diabetic encephalopathy [3] Methods for in vivo quantification and localization of changes in blood-brain barrier permeability are needed to understand and diagnose the early onset of vascular dementia with type 2 diabetes.

*Correspondence: c.ferris@northeastern.edu

${ }^{1}$ Center for Translational Neurolmaging, Northeastern University, Boston, MA, USA

Full list of author information is available at the end of the article
Imaging the subtle changes in blood-brain permeability is not possible with standard imaging protocols but can be assessed with dynamic contrast enhanced (DCE) MRI [4]. However, dynamic contrast enhanced MRI has several limitations. The concentration versus time curve for gadolinium-based contrast agent is typically $15-30 \%$ inaccurate; therefore, DCE-MRI has not proven useful clinically [5]. It is also difficult to model the effects of contrast agent on both $\mathrm{T} 2 *$ and $\mathrm{T} 1$ given the short acquisition time, and strong dependence on microstructural properties such as vessel size, tortuosity and orientation. These and other methodological issues with the use of DCE-MRI for blood-brain barrier permeability have resulted in significant differences in the reported rates of leakage [5].

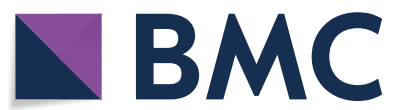

(c) The Author(s) 2020. This article is licensed under a Creative Commons Attribution 4.0 International License, which permits use, sharing, adaptation, distribution and reproduction in any medium or format, as long as you give appropriate credit to the original author(s) and the source, provide a link to the Creative Commons licence, and indicate if changes were made. The images or other third party material in this article are included in the article's Creative Commons licence, unless indicated otherwise in a credit line to the material. If material is not included in the article's Creative Commons licence and your intended use is not permitted by statutory regulation or exceeds the permitted use, you will need to obtain permission directly from the copyright holder. To view a copy of this licence, visit http://creativeco mmons.org/licenses/by/4.0/. The Creative Commons Public Domain Dedication waiver (http://creativecommons.org/publicdomain/ zero/1.0/) applies to the data made available in this article, unless otherwise stated in a credit line to the data. 
To address this issue, a novel imaging modality, quantitative ultrashort time-to-echo, contrast enhanced (QUTE-CE) MRI [6] was used to study changes in blood-brain barrier in the BBZDR/Wor rat an inbred rat strain model of type 2 diabetes [7]. QUTE-CE MRI utilizes Ultrashort-Time-to-Echo (UTE) sequences with ferumoxytol, an FDA-approved superparamagnetic iron oxide nanoparticles (SPIONs) formula already used offlabel for human MRI, as a contrast agent to produce positive contrast angiograms with low error of quantification $[6,8]$.

\section{Research design and methods Animals}

This study used male Bio-Breeding Zucker diabetic rats (BBZDR/Wor rats) $(n=8)$ and age-matched non-diabetic BBDR littermates $(n=7)$. The founding population was established by Biomere (Worcester, MA). The company decided to retire the breeding line and made a gift of their last animals to the Center for Translational NeuroImaging. The obese male BBZDR/Wor rat spontaneously develops type 2 diabetes at approximately 10 weeks of age $(\sim 100 \%)$ when fed standard rat chow. BBZDR/ Wor diabetic rat displays all clinical symptoms typically associated with type 2 diabetes including dyslipidemia, hyperglycemia, insulin resistance, and hypertension [7],

Rats were maintained on a $12 \mathrm{~h}: 12 \mathrm{~h}$ light-dark cycle with a light on at 07:00 h, allowed access to food and water ad libitum and were treated with intraperitoneal injections of saline at indications of weight loss. All animal experiments were conducted in accordance with the Northeastern University Division of Laboratory Animal Medicine and Institutional Animal Care and Use Committee. (https://academic.oup.com/ilarjournal/artic le/45/3/292/704910).

Access to rats was dependent upon the breeding schedule and resulting genotypes. This required we run two separate imaging studies, each with four rats from each genotype, separated by 6 months.

\section{Imaging}

Studies were done on a Bruker Biospec $7.0 \mathrm{~T} / 20 \mathrm{~cm}$ USR horizontal magnet (Bruker, Billerica, MA, USA) and a 20-G/cm magnetic field gradient insert $(\mathrm{ID}=12 \mathrm{~cm})$ capable of a $120 \mu \mathrm{s}$ rise time. Radio frequency signals were sent and received with a quadrature volume coil built into the rat restrainer (Animal Imaging Research, Holden, Massachusetts). All rats imaged under $1-2 \%$ isoflurane while keeping a respiratory rate of $40-50$ breadths $/ \mathrm{min}$. At the beginning of each imaging session, a high-resolution anatomical data set was collected using the RARE pulse sequence with following parameters, 35 slice of $0.7 \mathrm{~mm}$ thickness; field of view $3 \mathrm{~cm} ; 256 \times 256$; repetition time [TR] $3900 \mathrm{~ms}$; effective echo time [TE] $48 \mathrm{~ms}$; number of excitations $3 ; 6$ min $14 \mathrm{~s}$ acquisition time.

Rats were imaged prior to and following an i.v. bolus of $6 \mathrm{mg} / \mathrm{ml} \mathrm{Fe}$ of Ferumoxytol. The injected volume was tailored for each rat (assuming 7\% blood by body weight) to produce a starting blood concentration of $200 \mu \mathrm{g} / \mathrm{ml} \mathrm{Fe}(2 \times$ the clinical dose approved for use in humans). The QUTE-CE MRI image parameters of $\mathrm{TE}=13 \mu \mathrm{s}, \mathrm{TR}=4 \mathrm{~ms}$, and flip angle $=20^{\circ}$ utilized a high radio frequency pulse bandwidth of $200 \mathrm{kHz}$. Therefore, the pulse duration was short $(6.4 \mu \mathrm{s}) \mathrm{com}$ pared to the T2 of the approximate ferumoxytol concentration $(4.58 \mathrm{~ms}$ for $3.58 \mathrm{mM}$, i.e. $200 \mu \mathrm{g} / \mathrm{ml}$ to minimize signal blur and reduce the probability for a curved trajectory of the magnetization vector Mz. A $3 \times 3 \times 3 \mathrm{~cm}^{3}$ field-of-view was used with a matrix mesh size of $180 \times 180 \times 180$ to produce $167 \mu \mathrm{m}$ isotropic resolution.

Images were motion-corrected, aligned spatially, and resliced using MATLAB SPM12 toolbox developed at UCL (https://www.fil.ion.ucl.ac.uk/spm/). The precontrast UTE images were set as the baseline. For each rat in each imaging session, the voxel wise percentage change of signal intensity was calculated as (post-conbaseline)/(blood intensity change) ${ }^{*} 100 \%$ as described in our previous work [10], where blood intensity change is a normalization factor calculated by the postcon blood signal intensity minus baseline blood signal intensity. A 173-region rat brain atlas (Ekam Solutions LLC, Boston, MA, US) was fit to T2-weighted RARE anatomical data set for each rat data set taken at each imaging session, using software developed at Northeastern University Center for Translational Neuroimaging (CTNI), considering the variations in brain size and positions. The fitted atlas was transferred to UTE imaging. Once the images were co-registered to the atlas, custom MATLAB code was used to mask individual brain regions for ferumoxytol measurement. Post contrast UTE images are shown for a control and diabetic rat in Additional file 1: Figure S1.

Mode of percentage change distribution for each of the 173 brain areas for control and BBZDR/Wor rats was statistically compared using a Wilcoxon rank-sum test with the alpha set at 0.05 . Data was analyzed by co-authors Cai and Kulkarni blind to the identity of the groups.

\section{Data and resource availability}

All data can be accessed through a link to Mendeley. DOI to follow. 
Table 1 Brain areas that have significantly greater blood-brain barrier permeability in the diabetic BBZDR/Wor rat as compared to wild type controls

\begin{tabular}{|c|c|c|c|c|c|c|}
\hline \multirow[t]{2}{*}{ Brain area } & \multicolumn{2}{|c|}{ Control } & & \multicolumn{2}{|c|}{ Diabetes } & \multirow[t]{2}{*}{$P$ value } \\
\hline & Mean & SD & & Mean & SD & \\
\hline Parafascicular thalamic nucleus & 0.03 & 0.00 & $<$ & 0.08 & 0.01 & 0.000 \\
\hline Visual $1 \mathrm{ctx}$ & 0.03 & 0.00 & $<$ & 0.08 & 0.01 & 0.000 \\
\hline Entorhinal ctx & 0.03 & 0.01 & $<$ & 0.09 & 0.01 & 0.000 \\
\hline Dentate gyrus ventral & 0.03 & 0.01 & $<$ & 0.10 & 0.01 & 0.000 \\
\hline Medial geniculate & 0.03 & 0.00 & $<$ & 0.11 & 0.01 & 0.000 \\
\hline Medial dorsal thalamic nucleus & 0.02 & 0.00 & $<$ & 0.08 & 0.01 & 0.000 \\
\hline Visual $2 \mathrm{ctx}$ & 0.03 & 0.00 & $<$ & 0.09 & 0.01 & 0.000 \\
\hline Vuditory ctx & 0.03 & 0.00 & $<$ & 0.08 & 0.01 & 0.000 \\
\hline Ventral posteriolateral thalamic nucleus & 0.03 & 0.00 & $<$ & 0.07 & 0.01 & 0.000 \\
\hline Triangular septal nucleus & 0.02 & 0.01 & $<$ & 0.07 & 0.01 & 0.000 \\
\hline Bed nucleus stria terminalis & 0.01 & 0.00 & $<$ & 0.05 & 0.01 & 0.000 \\
\hline Inferior colliculus & 0.04 & 0.01 & $<$ & 0.11 & 0.02 & 0.000 \\
\hline Posterior thalamic nucleus & 0.03 & 0.00 & $<$ & 0.07 & 0.01 & 0.000 \\
\hline Dorsal lateral striatum & 0.02 & 0.00 & $<$ & 0.06 & 0.01 & 0.000 \\
\hline Lateral posterior thalamic nucleus & 0.03 & 0.01 & $<$ & 0.09 & 0.01 & 0.000 \\
\hline Reticular nucleus & 0.03 & 0.00 & $<$ & 0.07 & 0.01 & 0.000 \\
\hline CA1 dorsal & 0.03 & 0.00 & $<$ & 0.06 & 0.01 & 0.000 \\
\hline Dentate gyrus dorsal & 0.03 & 0.00 & $<$ & 0.08 & 0.01 & 0.000 \\
\hline Central amygdaloid nucleus & 0.01 & 0.01 & $<$ & 0.05 & 0.01 & 0.000 \\
\hline Ventral lateral striatum & 0.02 & 0.00 & $<$ & 0.06 & 0.01 & 0.000 \\
\hline Reuniens nucleus & 0.03 & 0.00 & $<$ & 0.07 & 0.01 & 0.000 \\
\hline Globus pallidus & 0.02 & 0.00 & $<$ & 0.05 & 0.01 & 0.000 \\
\hline Lateral geniculate & 0.04 & 0.01 & $<$ & 0.09 & 0.01 & 0.000 \\
\hline Dorsal medial striatum & 0.02 & 0.00 & $<$ & 0.06 & 0.01 & 0.000 \\
\hline Paraventricular nucleus & 0.03 & 0.00 & $<$ & 0.07 & 0.01 & 0.000 \\
\hline Retrosplenial caudal ctx & 0.03 & 0.01 & $<$ & 0.12 & 0.02 & 0.000 \\
\hline Lateral septal nucleus & 0.02 & 0.00 & $<$ & 0.06 & 0.01 & 0.000 \\
\hline CA2 & 0.03 & 0.00 & $<$ & 0.06 & 0.01 & 0.000 \\
\hline Ventrolateral thalamic nucleus & 0.03 & 0.00 & $<$ & 0.07 & 0.01 & 0.000 \\
\hline External plexiform layer & 0.07 & 0.01 & $<$ & 0.12 & 0.01 & 0.000 \\
\hline Periaqueductal gray thalamus & 0.04 & 0.00 & $<$ & 0.08 & 0.01 & 0.000 \\
\hline Temporal ctx & 0.03 & 0.01 & $<$ & 0.12 & 0.02 & 0.000 \\
\hline Ventral subiculum & 0.04 & 0.00 & $<$ & 0.08 & 0.01 & 0.000 \\
\hline Ventral posteriomedial thalamic nucleus & 0.03 & 0.00 & $<$ & 0.08 & 0.01 & 0.000 \\
\hline Basal amygdaloid nucleus & 0.01 & 0.01 & $<$ & 0.06 & 0.01 & 0.000 \\
\hline Ventromedial thalamic nucleus & 0.03 & 0.00 & $<$ & 0.08 & 0.01 & 0.000 \\
\hline Parietal ctx & 0.03 & 0.00 & $<$ & 0.06 & 0.01 & 0.000 \\
\hline Caudal piriform ctx & 0.03 & 0.00 & $<$ & 0.08 & 0.02 & 0.000 \\
\hline Medial amygdaloid nucleus & 0.03 & 0.01 & $<$ & 0.09 & 0.02 & 0.000 \\
\hline CA1 hippocampus ventral & 0.04 & 0.01 & $<$ & 0.08 & 0.01 & 0.000 \\
\hline Primary somatosensory ctx barrel field & 0.03 & 0.00 & $<$ & 0.07 & 0.01 & 0.000 \\
\hline Zona incerta & 0.04 & 0.01 & $<$ & 0.08 & 0.01 & 0.000 \\
\hline Primary somatosensory ctx forelimb & 0.02 & 0.00 & $<$ & 0.06 & 0.01 & 0.000 \\
\hline Granular cell layer & 0.06 & 0.01 & $<$ & 0.10 & 0.01 & 0.000 \\
\hline Habenula nucleus & 0.06 & 0.01 & $<$ & 0.15 & 0.03 & 0.000 \\
\hline Primary somatosensory ctx trunk & 0.03 & 0.00 & $<$ & 0.06 & 0.01 & 0.000 \\
\hline
\end{tabular}


Table 1 (continued)

\begin{tabular}{|c|c|c|c|c|c|c|}
\hline \multicolumn{7}{|c|}{ Areas with significant changes in blood-brain barrier permeability } \\
\hline \multirow[t]{2}{*}{ Brain area } & \multicolumn{2}{|c|}{ Control } & & \multicolumn{2}{|c|}{ Diabetes } & \multirow[t]{2}{*}{$P$ value } \\
\hline & Mean & SD & & Mean & SD & \\
\hline Lateral hypothalamus & 0.04 & 0.00 & $<$ & 0.08 & 0.02 & 0.000 \\
\hline Primary somatosensory ctx shoulder & 0.02 & 0.01 & $<$ & 0.06 & 0.01 & 0.000 \\
\hline Ventral medial striatum & 0.02 & 0.00 & $<$ & 0.05 & 0.01 & 0.000 \\
\hline Glomerular layer & 0.09 & 0.01 & $<$ & 0.14 & 0.02 & 0.000 \\
\hline Prerubral field & 0.04 & 0.01 & $<$ & 0.08 & 0.01 & 0.000 \\
\hline Extended amygdala & 0.02 & 0.00 & $<$ & 0.05 & 0.01 & 0.000 \\
\hline Anterior hypothalamic area & 0.02 & 0.00 & $<$ & 0.06 & 0.01 & 0.000 \\
\hline Primary motor ctx & 0.02 & 0.00 & $<$ & 0.06 & 0.01 & 0.000 \\
\hline Secondary somatosensory ctx & 0.03 & 0.00 & $<$ & 0.07 & 0.01 & 0.000 \\
\hline Intercalated amygdaloid nucleus & 0.01 & 0.01 & $<$ & 0.07 & 0.01 & 0.000 \\
\hline Primary somatosensory ctx upper lip & 0.03 & 0.00 & $<$ & 0.07 & 0.01 & 0.000 \\
\hline White matter rostral & 0.03 & 0.00 & $<$ & 0.06 & 0.01 & 0.000 \\
\hline CA3 dorsal & 0.03 & 0.00 & $<$ & 0.06 & 0.01 & 0.000 \\
\hline Posterior hypothalamic area & 0.04 & 0.01 & $<$ & 0.10 & 0.02 & 0.000 \\
\hline Central medial thalamic nucleus & 0.04 & 0.00 & $<$ & 0.07 & 0.01 & 0.000 \\
\hline Dorsal raphe & 0.04 & 0.01 & $<$ & 0.08 & 0.01 & 0.000 \\
\hline Supramammillary nucleus & 0.05 & 0.01 & $<$ & 0.15 & 0.04 & 0.000 \\
\hline Primary somatosensory ctx hindlimb & 0.03 & 0.00 & $<$ & 0.06 & 0.01 & 0.000 \\
\hline Ventral anterior thalamic nucleus & 0.03 & 0.01 & $<$ & 0.07 & 0.01 & 0.000 \\
\hline Lateral amygdaloid nucleus & 0.03 & 0.01 & $<$ & 0.07 & 0.01 & 0.000 \\
\hline Claustrum & 0.02 & 0.00 & $<$ & 0.05 & 0.01 & 0.000 \\
\hline Perirhinal ctx & 0.05 & 0.01 & $<$ & 0.12 & 0.02 & 0.000 \\
\hline Lateral dorsal thalamic nucleus & 0.03 & 0.01 & $<$ & 0.06 & 0.01 & 0.000 \\
\hline Dorsal medial nucleus & 0.03 & 0.01 & $<$ & 0.07 & 0.01 & 0.000 \\
\hline Ectorhinal ctx & 0.04 & 0.01 & $<$ & 0.15 & 0.05 & 0.000 \\
\hline Olivary nucleus & 0.05 & 0.01 & $<$ & 0.09 & 0.02 & 0.000 \\
\hline Copula of the pyramis & 0.07 & 0.01 & $<$ & 0.11 & 0.02 & 0.000 \\
\hline Motor trigeminal nucleus & 0.04 & 0.01 & $<$ & 0.07 & 0.01 & 0.000 \\
\hline Paramedian lobule & 0.06 & 0.00 & $<$ & 0.08 & 0.01 & 0.000 \\
\hline Solitary tract nucleus & 0.03 & 0.01 & $<$ & 0.06 & 0.01 & 0.000 \\
\hline Parvicellular reticular areas & 0.04 & 0.00 & $<$ & 0.06 & 0.01 & 0.000 \\
\hline Precuniform nucleus & 0.04 & 0.01 & $<$ & 0.07 & 0.01 & 0.000 \\
\hline Anterior cingulate area & 0.03 & 0.00 & $<$ & 0.08 & 0.02 & 0.000 \\
\hline Cortical amygdaloid nucleus & 0.05 & 0.01 & $<$ & 0.10 & 0.02 & 0.000 \\
\hline Primary somatosensory ctx jaw & 0.03 & 0.01 & $<$ & 0.06 & 0.01 & 0.000 \\
\hline Parabrachial nucleus & 0.05 & 0.01 & $<$ & 0.08 & 0.01 & 0.000 \\
\hline Principal sensory nucleus trigeminal & 0.05 & 0.00 & $<$ & 0.07 & 0.01 & 0.000 \\
\hline Sub coeruleus nucleus & 0.04 & 0.00 & $<$ & 0.06 & 0.01 & 0.000 \\
\hline White matter caudal & 0.04 & 0.00 & $<$ & 0.07 & 0.02 & 0.000 \\
\hline Endopiriform nucleus & 0.02 & 0.01 & $<$ & 0.05 & 0.01 & 0.000 \\
\hline Reticular nucleus midbrain & 0.04 & 0.01 & $<$ & 0.08 & 0.02 & 0.000 \\
\hline Anterior thalamic nuclei & 0.03 & 0.01 & $<$ & 0.07 & 0.02 & 0.000 \\
\hline Accumbens core & 0.02 & 0.01 & $<$ & 0.05 & 0.02 & 0.000 \\
\hline Prelimbic ctx & 0.03 & 0.00 & $<$ & 0.06 & 0.02 & 0.000 \\
\hline 7th cerebellar lobule & 0.03 & 0.01 & $<$ & 0.06 & 0.01 & 0.000 \\
\hline CA3 hippocampus ventral & 0.04 & 0.01 & $<$ & 0.08 & 0.02 & 0.000 \\
\hline Ventral medial nucleus & 0.03 & 0.01 & $<$ & 0.08 & 0.03 & 0.000 \\
\hline
\end{tabular}


Table 1 (continued)

\begin{tabular}{|c|c|c|c|c|c|c|}
\hline \multicolumn{7}{|c|}{ Areas with significant changes in blood-brain barrier permeability } \\
\hline \multirow[t]{2}{*}{ Brain area } & \multicolumn{2}{|c|}{ Control } & & \multicolumn{2}{|c|}{ Diabetes } & \multirow[t]{2}{*}{$P$ value } \\
\hline & Mean & SD & & Mean & SD & \\
\hline Dorsal paragigantocellularis & 0.03 & 0.01 & $<$ & 0.05 & 0.01 & 0.000 \\
\hline Median raphe nucleus & 0.04 & 0.01 & $<$ & 0.06 & 0.01 & 0.000 \\
\hline Pedunculopontine tegmental area & 0.04 & 0.01 & $<$ & 0.07 & 0.02 & 0.000 \\
\hline Secondary motor ctx & 0.03 & 0.01 & $<$ & 0.07 & 0.02 & 0.000 \\
\hline Central gray & 0.05 & 0.00 & $<$ & 0.08 & 0.01 & 0.000 \\
\hline Retrosplenial rostral ctx & 0.05 & 0.01 & $<$ & 0.11 & 0.03 & 0.001 \\
\hline Subthalamic nucleus & 0.07 & 0.01 & $<$ & 0.11 & 0.02 & 0.001 \\
\hline Medial preoptic area & 0.02 & 0.01 & $<$ & 0.05 & 0.01 & 0.001 \\
\hline Medial septum & 0.03 & 0.01 & $<$ & 0.06 & 0.01 & 0.001 \\
\hline Gigantocellularis reticular nucleus pons & 0.03 & 0.00 & $<$ & 0.05 & 0.01 & 0.001 \\
\hline Superior colliculus & 0.04 & 0.01 & $<$ & 0.07 & 0.02 & 0.001 \\
\hline Subiculum dorsal & 0.04 & 0.00 & $<$ & 0.06 & 0.01 & 0.001 \\
\hline Lateral preoptic area & 0.02 & 0.01 & $<$ & 0.05 & 0.02 & 0.001 \\
\hline Magnocellular preoptic nucleus & 0.03 & 0.01 & $<$ & 0.08 & 0.03 & 0.001 \\
\hline Dorsomedial tegmental area & 0.04 & 0.01 & $<$ & 0.06 & 0.02 & 0.001 \\
\hline Neural lobe pituitary & 0.14 & 0.05 & $<$ & 0.26 & 0.06 & 0.001 \\
\hline Medial cerebellar nucleus fastigial & 0.06 & 0.01 & $<$ & 0.08 & 0.01 & 0.001 \\
\hline Substantia nigra compacta & 0.05 & 0.01 & $<$ & 0.10 & 0.03 & 0.001 \\
\hline 8th cerebellar lobule & 0.04 & 0.01 & $<$ & 0.06 & 0.01 & 0.001 \\
\hline Medial mammillary nucleus & 0.07 & 0.04 & $<$ & 0.20 & 0.08 & 0.002 \\
\hline Pontine reticular nucleus caudal & 0.03 & 0.00 & $<$ & 0.05 & 0.01 & 0.002 \\
\hline Flocculus cerebellum & 0.05 & 0.01 & $<$ & 0.08 & 0.02 & 0.002 \\
\hline Substantia nigra reticularis & 0.07 & 0.02 & $<$ & 0.12 & 0.03 & 0.002 \\
\hline Supraoptic nucleus & 0.05 & 0.01 & $<$ & 0.08 & 0.02 & 0.002 \\
\hline Reticulotegmental nucleus & 0.03 & 0.01 & $<$ & 0.05 & 0.01 & 0.003 \\
\hline Anterior lobe pituitary & 0.17 & 0.02 & $<$ & 0.28 & 0.07 & 0.003 \\
\hline Accumbens shell & 0.03 & 0.01 & $<$ & 0.06 & 0.02 & 0.003 \\
\hline Inferior olivary complex & 0.05 & 0.00 & $<$ & 0.07 & 0.02 & 0.003 \\
\hline 10th cerebellar lobule & 0.07 & 0.01 & $<$ & 0.09 & 0.01 & 0.003 \\
\hline Infralimbic ctx & 0.03 & 0.01 & $<$ & 0.07 & 0.02 & 0.003 \\
\hline Cochlear nucleus & 0.06 & 0.01 & $<$ & 0.08 & 0.02 & 0.004 \\
\hline Premammillary nucleus & 0.05 & 0.02 & $<$ & 0.11 & 0.04 & 0.004 \\
\hline Insular ctx & 0.04 & 0.01 & $<$ & 0.07 & 0.02 & 0.004 \\
\hline Red nucleus & 0.05 & 0.01 & $<$ & 0.08 & 0.02 & 0.004 \\
\hline Suprachiasmatic nucleus & 0.01 & 0.02 & $<$ & 0.05 & 0.02 & 0.005 \\
\hline Root of trigeminal nerve & 0.05 & 0.00 & $<$ & 0.07 & 0.02 & 0.005 \\
\hline Interposed nucleus & 0.06 & 0.01 & $<$ & 0.08 & 0.01 & 0.006 \\
\hline Vestibular nucleus & 0.05 & 0.01 & $<$ & 0.06 & 0.01 & 0.006 \\
\hline 9th cerebellar lobule & 0.05 & 0.01 & $<$ & 0.07 & 0.01 & 0.007 \\
\hline 2nd cerebellar lobule & 0.07 & 0.01 & $<$ & 0.10 & 0.02 & 0.007 \\
\hline Pontine reticular nucleus oral & 0.04 & 0.01 & $<$ & 0.05 & 0.01 & 0.008 \\
\hline Retrochiasmatic nucleus & 0.05 & 0.04 & $<$ & 0.13 & 0.06 & 0.009 \\
\hline Anterior pretectal nucleus & 0.03 & 0.00 & $<$ & 0.07 & 0.03 & 0.009 \\
\hline Trapezoid body & 0.03 & 0.01 & $<$ & 0.06 & 0.02 & 0.010 \\
\hline Facial nucleus & 0.05 & 0.01 & $<$ & 0.07 & 0.02 & 0.010 \\
\hline Raphe obscurus nucleus & 0.03 & 0.01 & $<$ & 0.04 & 0.01 & 0.011 \\
\hline Ventral pallidum & 0.04 & 0.01 & $<$ & 0.07 & 0.02 & 0.011 \\
\hline
\end{tabular}


Table 1 (continued)

\begin{tabular}{|c|c|c|c|c|c|c|}
\hline \multicolumn{7}{|c|}{ Areas with significant changes in blood-brain barrier permeability } \\
\hline \multirow[t]{2}{*}{ Brain area } & \multicolumn{2}{|c|}{ Control } & & \multicolumn{2}{|c|}{ Diabetes } & \multirow[t]{2}{*}{$P$ value } \\
\hline & Mean & SD & & Mean & SD & \\
\hline Raphe linear & 0.06 & 0.01 & $<$ & 0.08 & 0.02 & 0.012 \\
\hline Periolivary nucleus & 0.05 & 0.02 & $<$ & 0.08 & 0.03 & 0.019 \\
\hline Dentate $n$. cerebellum & 0.05 & 0.01 & $<$ & 0.06 & 0.02 & 0.019 \\
\hline Arcuate nucleus & 0.07 & 0.04 & $<$ & 0.13 & 0.06 & 0.023 \\
\hline Substantia innominata & 0.05 & 0.02 & $<$ & 0.09 & 0.03 & 0.024 \\
\hline Paraflocculus cerebellum & 0.06 & 0.01 & $<$ & 0.07 & 0.02 & 0.026 \\
\hline Anterior amygdaloid nucleus & 0.03 & 0.01 & $<$ & 0.05 & 0.03 & 0.035 \\
\hline
\end{tabular}

Areas are ranked in order of their significance $(a<0.05)$. False detection rate $(a=0.17)$

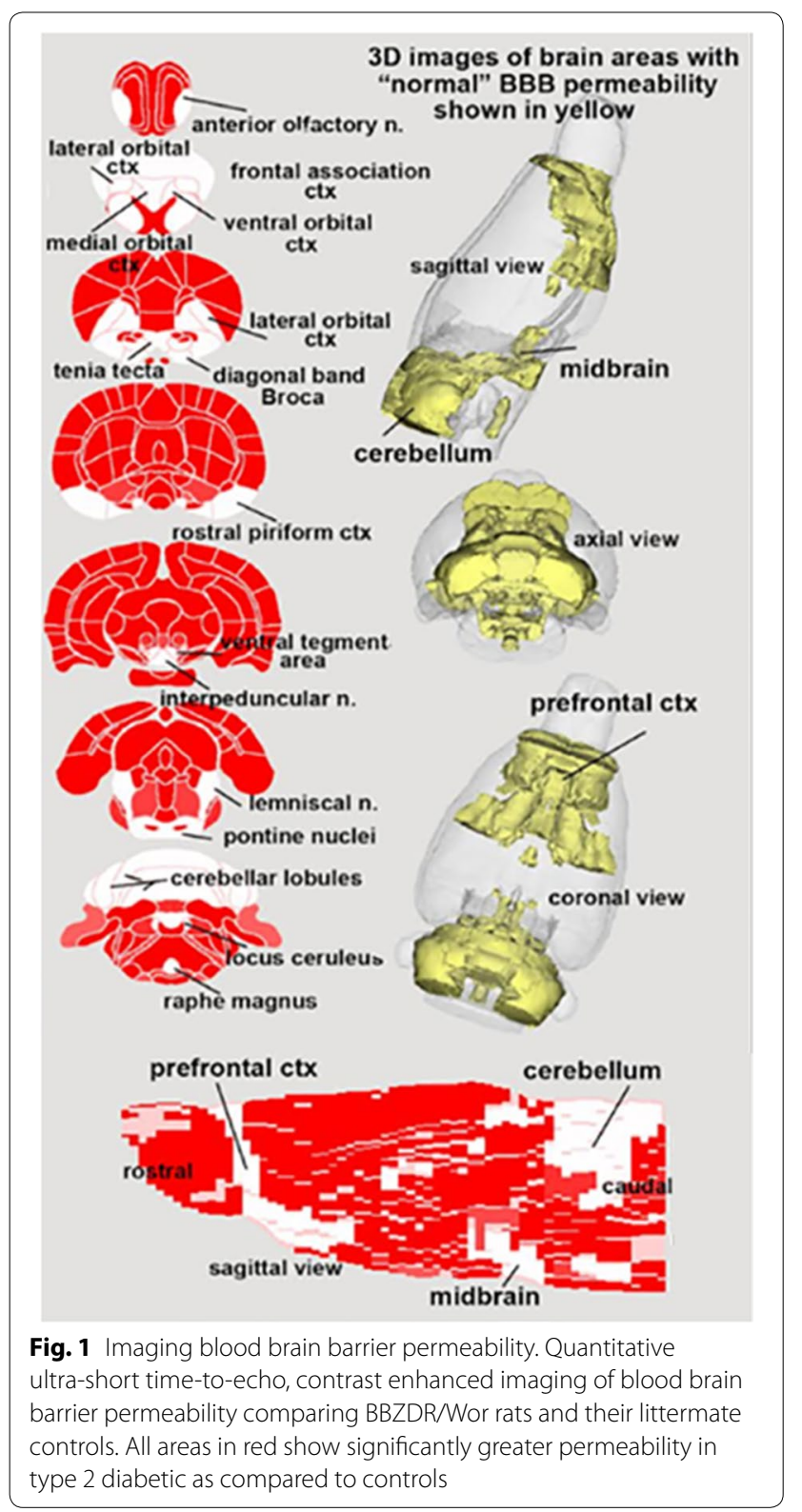

\section{Results}

Table 1 shows all the brain areas (147/173) that were significantly different $(\alpha \mathrm{p}<0.05)$ in blood-brain barrier permeability between BBZDR/Wor rats and their littermate controls. Note in all cases BBZDR/Wor rats showed greater permeability. The location of these areas can be are visualized in the surrounding $2 \mathrm{D}$ and $3 \mathrm{D}$ images generated with the rat MRI atlas shown in Fig. 1. All areas in red in the $2 \mathrm{D}$ representations show significantly greater blood-brain barrier permeability in the BBZDR/Wor rats as compared to controls. Table 2 shows all brain areas $(26 / 173)$ that were not significantly different in bloodbrain barrier permeability between BBZDR/Wor rats and their littermate controls. These areas shown in white are localized to the prefrontal ctx, midbrain and cerebellum. These nonaffected areas are coalesced into 3D volumes and pictured in the glass brain in yellow.

\section{Discussion}

QUTE-CE MRI, was developed as a quantitative vascular biomarker [6]. Ferumoxytol $\left(\right.$ Feraheme $^{\mathrm{TM}}$ ) MRI with optimized 3D Ultra-Short Time-to-echo (UTE) Pulse Sequences produces angiographic images unparalleled to time-of-flight imaging or gadolinium-based first-pass imaging. The contrast agent is ferumoxytol, an ultrasmall superparamagnetic iron oxide nanoparticle with a dextran coating. Since the size exceeds the cutoff $(\sim 6 \mathrm{~nm})$ for glomerular filtration, ferumoxytol is not cleared by the kidney, and instead is an excellent blood pool contrast agent with a long intravascular half-life of $\sim 15 \mathrm{~h}$ [9]. Numerous clinical MRI studies using ferumoxytol have been conducted in children and adults, demonstrating no major adverse effects, thus QUTE-CE can be readily used in the clinic to study blood-brain barrier permeability [10]. We recently published a study mapping the absolute physiological cerebral blood volume (CBV) of the awake rat brain, including measurements of microvasculature 
Table 2 Brain areas that show no significant differences in blood-brain barrier permeability in the diabetic BBZDR/Wor rat as compared to wild type controls

\begin{tabular}{|c|c|c|c|c|c|c|}
\hline \multicolumn{7}{|c|}{ Areas with nonsignificant changes in blood-brain barrier permeability } \\
\hline \multirow[t]{2}{*}{ Brain area } & \multicolumn{2}{|c|}{ Control } & & \multicolumn{2}{|c|}{ Diabetes } & \multirow[b]{2}{*}{$P$ value } \\
\hline & Mean & SD & & Mean & SD & \\
\hline Olfactory tubercles & 0.07 & 0.02 & $<$ & 0.10 & 0.04 & 0.067 \\
\hline Medial pretectal area & 0.00 & 0.16 & $<$ & 0.17 & 0.18 & 0.070 \\
\hline Raphe magnus & 0.03 & 0.01 & $<$ & 0.05 & 0.02 & 0.088 \\
\hline Paraventricular nucleus & 0.06 & 0.02 & $<$ & 0.09 & 0.05 & 0.091 \\
\hline 3rd cerebellar lobule & 0.07 & 0.01 & $<$ & 0.09 & 0.03 & 0.110 \\
\hline Ventral tegmental area & 0.07 & 0.02 & $<$ & 0.11 & 0.06 & 0.130 \\
\hline Rostral piriform ctx & 0.05 & 0.02 & $<$ & 0.07 & 0.03 & 0.143 \\
\hline Locus coeruleus & 0.07 & 0.01 & $<$ & 0.08 & 0.01 & 0.146 \\
\hline Diagonal band of Broca & 0.04 & 0.02 & $<$ & 0.06 & 0.03 & 0.157 \\
\hline Ventral orbital ctx & 0.04 & 0.01 & $<$ & 0.06 & 0.03 & 0.166 \\
\hline 6th cerebellar lobule & 0.04 & 0.01 & $<$ & 0.05 & 0.01 & 0.200 \\
\hline Lateral orbital ctx & 0.04 & 0.01 & $<$ & 0.06 & 0.03 & 0.285 \\
\hline Anterior olfactory nucleus & 0.05 & 0.01 & $<$ & 0.07 & 0.04 & 0.289 \\
\hline Tenia tecta ctx & 0.06 & 0.02 & $<$ & 0.09 & 0.06 & 0.290 \\
\hline 1st cerebellar lobule & 0.05 & 0.01 & $<$ & 0.06 & 0.02 & 0.353 \\
\hline Simple lobule cerebellum & 0.09 & 0.01 & $>$ & 0.07 & 0.05 & 0.423 \\
\hline Pontine nuclei & 0.05 & 0.03 & $<$ & 0.07 & 0.05 & 0.451 \\
\hline Pineal gland & 0.86 & 0.04 & $<$ & 0.90 & 0.12 & 0.458 \\
\hline Interpeduncular nucleus & 0.09 & 0.04 & $<$ & 0.11 & 0.08 & 0.522 \\
\hline Medial orbital ctx & 0.09 & 0.01 & $>$ & 0.08 & 0.05 & 0.526 \\
\hline Crus 2 of ansiform lobule & 0.06 & 0.00 & & 0.06 & 0.01 & 0.604 \\
\hline Lemniscal nucleus & 0.06 & 0.02 & $<$ & 0.07 & 0.03 & 0.611 \\
\hline Frontal association ctx & 0.08 & 0.01 & & 0.08 & 0.04 & 0.658 \\
\hline Crus 1 of ansiform lobule & 0.06 & 0.01 & $>$ & 0.05 & 0.02 & 0.790 \\
\hline 4th cerebellar lobule & 0.07 & 0.01 & & 0.07 & 0.04 & 0.809 \\
\hline 5th cerebellar lobule & 0.07 & 0.01 & $>$ & 0.06 & 0.04 & 0.856 \\
\hline
\end{tabular}

Areas are ranked in order of their $P$ values

density and vascular functional reserve [8]. QUTE-CE MRI can be used for identifying hyper- or hypo-vascularization, small vessel density, blood-brain barrier permeability and vascular reserve and vascular responsivity to $\mathrm{CO} 2$ challenge at the individual voxel and regional levels using our rat 3D MRI atlas. As demonstrated in this study with the BBZDR/Wor rats, a preclinical model of type 2 diabetes, this imaging technology could be used to diagnose and evaluate blood brain permeability and disease progression in diabetic encephalopathy in the clinic.

\section{Limitations and future directions}

As a pilot study with a small population of rats there were several limitations: (1) Females were not studied. Unfortunately, only males develop diabetes in the BBZDR/Wor strain of rats [7]. (2) While the bloodbrain permeability was pervasive in this late-stage model of diabetes and not unexpected, postmortem histology would have confirmed the capillary pathology and helped to understand why areas like the cerebellum and midbrain were spared. (3) In the future, a thorough comparison between DCE and QUTE-CE should be done to provide quantitative data on the differences and similarities between both imaging techniques. (4) More common rat models of T2DB should be tested like the Goto-Kakizaki GK rat [11] or high-fat diet, streptozotocin treated Wistar rat (HFD/STZ) [12].

\section{Conclusion}

Small vessel disease as assessed by permeability in the blood-brain barrier in type 2 diabetes is pervasive and includes much of the brain. The increase in bloodbrain barrier permeability is a likely contributing factor to diabetic encephalopathy and dementia. 


\section{Supplementary information}

Supplementary information accompanies this paper at https://doi. org/10.1186/s12967-020-02428-3.

Additional file 1: Figure S1. Shown are raw data from a control and diabetic rat following ferumoxytol injection. The normalized UTE signal is registered to the original anatomy.

\section{Abbreviations}

QUTE-CE: Quantitative ultrashort time-to-echo, contrast enhanced; BBB: Blood brain barrier; CBV: Cerebral blood volume; DCE: Dynamic contrast enhanced; BBZDR: Male Bio-Breeding Zucker diabetic rats; TR: Repetition time; TE: Echo time; RARE: Rapid acquisition with relaxation enhancement.

\section{Acknowledgements}

We thank Biomere (Worcester, Massachusetts, USA) for providing the BBZDR/ Wor rats and their age-matched non-diabetic BBDR littermates for these studies.

\section{Authors' contributions}

All of the authors have contributed substantially to the manuscript. Concept, drafting and interpretation-CFF, JQ. Execution and analysis-JQ, PK, CML, KFGR. All authors read and approved the final manuscript.

\section{Funding}

Not applicable.

\section{Availability of data and materials}

All data can be accessed through a link to Mendeley. DOI to follow.

\section{Ethics approval and consent to participate}

Not applicable to humans. All animal experiments were conducted in accordance with the Northeastern University Division of Laboratory Animal Medicine and Institutional Animal Care and Use Committee.

\section{Consent for publication}

Not applicable.

\section{Competing interests}

CFF has a financial interest in Animal Imaging Research, the company that makes the RF electronics and holders for animal imaging.

\section{Author details}

${ }^{1}$ Center for Translational Neurolmaging, Northeastern University, Boston, MA USA. ${ }^{2}$ Department of Psychology, Northeastern University, 360 Huntington Ave, Boston, MA 02115-5000, USA.

Received: 8 March 2020 Accepted: 18 June 2020

Published online: 24 June 2020

\section{References}

1. Pasquier F, Boulogne A, Leys D, Fontaine P. Diabetes mellitus and dementia. Diabetes Metab. 2006. https://doi.org/10.1016/s1262-3636(07)70298 $-7$

2. Allen $\mathrm{CL}$, Bayraktutan U. Antioxidants attenuate hyperglycaemiamediated brain endothelial cell dysfunction and blood-brain barrier hyperpermeability. Diabetes Obes Metab. 2009. https://doi.org/10.111 1/j.1463-1326.2008.00987.x.

3. Bogush $M$, Heldt NA, Persidsky Y. Blood brain barrier injury in diabetes: unrecognized effects on brain and cognition. J Neuroimmune Pharmacol. 2017. https://doi.org/10.1007/s11481-017-9752-7.

4. Starr JM, Wardlaw J, Ferguson K, MacLullich A, Deary IJ, Marshall I. Increased blood-brain barrier permeability in type II diabetes demonstrated by gadolinium magnetic resonance imaging. J Neurol Neurosurg Psychiatry. 2003. https://doi.org/10.1136/jnnp.74.1.70.

5. Raja R, Rosenberg GA, Caprihan A. MRI measurements of Blood-Brain Barrier function in dementia: a review of recent studies. Neuropharmacology. 2018. https://doi.org/10.1016/j.neuropharm.2017.10.034.

6. Gharagouzloo CA, McMahon PN, Sridhar S. Quantitative contrastenhanced MRI with superparamagnetic nanoparticles using ultrashort time-to-echo pulse sequences. Magn Reson Med. 2015. https://doi. org/10.1002/mrm.25426.

7. Tirabassi RS, Flanagan JF, Wu T, Kislauskis EH, Birckbichler PJ, Guberski DL. The BBZDR/Wor rat model for investigating the complications of type 2 diabetes mellitus. ILAR J. 2004. https://doi.org/10.1093/ilar.45.3.292.

8. Gharagouzloo CA, Timms L, Qiao J, Fang Z, Nneji J, Pandya A, Kulkarni P, van de Ven AL, Ferris C, Sridhar S. Quantitative vascular neuroimaging of the rat brain using superparamagnetic nanoparticles: New insights on vascular organization and brain function. Neurolmage. 2017. https://doi. org/10.1016/j.neuroimage.2017.09.003.

9. Bremerich J, Bilecen D, Reimer P. MR angiography with blood pool contrast agents. Eur Radiol. 2007. https://doi.org/10.1007/s00330-007-0712-0.

10. Muehe AM, Feng D, von Eyben R, Luna-Fineman S, Link MP, Muthig T, Huddleston AE, Neuwelt EA, Daldrup-Link HE. Safety report of ferumoxytol for magnetic resonance imaging in children and young adults. Invest Radiol. 2016. https://doi.org/10.1097/RLI.0000000000000230.

11. Kuwabara WMT, Panveloski-Costa AC, Yokota CNF, Pereira JNB, Filho JM, Torres RP, Hirabara SM, Curi R, Alba-Loureiro TC. Comparison of Goto-Kakizaki rats and high fat diet-induced obese rats: are they reliable models to study type 2 diabetes mellitus? PLoS ONE. 2017. https://doi.org/10.1371/ journal.pone.0189622

12. Ding G, Yan T, Chen J, Chopp M, Li L, Li Q, Cui C, Ning R, Jiang Q. Persistent cerebrovascular damage after stroke in type two diabetic rats measured by magnetic resonance imaging. Stroke. 2015. https://doi.org/10.1161/ STROKEAHA.114.007538.

\section{Publisher's Note}

Springer Nature remains neutral with regard to jurisdictional claims in published maps and institutional affiliations.
Ready to submit your research? Choose BMC and benefit from:

- fast, convenient online submission

- thorough peer review by experienced researchers in your field

- rapid publication on acceptance

- support for research data, including large and complex data types

- gold Open Access which fosters wider collaboration and increased citations

- maximum visibility for your research: over 100M website views per year

At BMC, research is always in progress.

Learn more biomedcentral.com/submissions 